Supplementary information for:

\title{
Immunoavidity-based Capture of Tumor Exosomes using Poly(amidoamine) Dendrimer Surfaces
}

Michael J. Poellmann ${ }^{1}$, Ashita Nair ${ }^{1}$, Jiyoon Bu ${ }^{1}$, Jack K. H. Kim ${ }^{1}$, Randall J. Kimple ${ }^{2,3}$, and

Seungpyo Hong ${ }^{1,3,4,5 *}$

${ }^{1}$ Pharmaceutical Sciences Division, School of Pharmacy, University of Wisconsin-Madison, WI 53705

${ }^{2}$ Department of Human Oncology, School of Medicine and Public Health, University of Wisconsin-Madison, WI 53705

${ }^{3}$ Carbone Cancer Center, University of Wisconsin-Madison, WI 53705

${ }^{4}$ Department of Biomedical Engineering, University of Wisconsin-Madison, WI 53706

${ }^{5}$ Yonsei Frontier Lab and Department of Pharmacy, Yonsei University, Seoul, KOREA 03722 
Supplementary information: Poellmann MJ et al, "Immunoavidity-Based Capture of Tumor Exosomes using Poly(amidoamine) Dendrimer Surfaces."

\section{Contents}

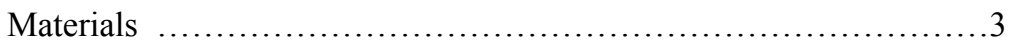

Nanoparticle Carboxylation ......................................

Figure S1 4

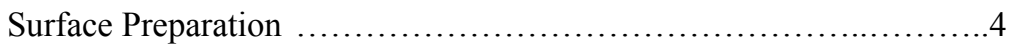

Contact Angle Measurements ....................................

$\begin{array}{ll}\text { Figure S2 } & 7\end{array}$

Roughness Measurements ....................................... 8

$\begin{array}{lr}\text { Figure S3 } & 8\end{array}$

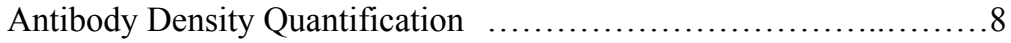

$\begin{array}{lr}\text { Figure S4 } & 9\end{array}$

Exosome Culture and Isolation ...................................... 9

$\begin{array}{lr}\text { Table S1 } & 10\end{array}$

$\begin{array}{ll}\text { Figure S5 } & 11\end{array}$

Capture and Quantification of In Vitro Exosomes....................11

Capture and Quantification of Exosomes in Donor Blood Samples....12

Table S2 13

AFM Force Spectroscopy ..........................................13

$\begin{array}{lr}\text { Figure S6 } & 15\end{array}$

Statistical Analysis ...................................................16 
Supplementary information: Poellmann MJ et al, "Immunoavidity-Based Capture of Tumor Exosomes using Poly(amidoamine) Dendrimer Surfaces."

\section{Materials}

Generation 7 (G7) poly(amidoamine) (PAMAM) dendrimers were obtained from Dendritech (Midland, MI) and all poly(ethylene glycol) (PEG) was obtained from JenKem (Plano, TX). Epoxide-functionalized glass surfaces were in the form of standard microscope slides from Tekdon (Myakka City, FL). Recombinant human epithelial cell adhesion molecule (EpCAM), antibodies targeting EpCAM (aEpCAM), epidermal growth factor receptor (aEGFR), and human epidermal growth factor receptor 2 (aHER2) were obtained from R\&D Systems (Minneapolis, MN), while aCD63, aCD81, aCD9, and aHSP70 were obtained from Santa Cruz Biotechnology (Dallas, TX). Sharp silicon nitride probes for imaging (SNL) were obtained from Bruker Probes (Camarillo, CA), while the TR400PB gold-coated probe was obtained from Oxford Instruments (Santa Barbara, CA). The lipophilic dye used to label exosomes was Vybrant DiO, obtained from Thermo Fisher Scientific (Waltham, MA). The SMCC crosslinker was supplied by CalBioChem (San Diego, CA), and all other reagents and human serum were supplied by Sigma Aldrich (St. Louis, MO).

\section{Nanoparticle Carboxylation}

Generation 7 PAMAM dendrimers at 5 wt.\% in methanol were purified 5 times with phosphate buffered saline (PBS) and 5 times with double distilled (DDI) water by centrifugal filtration through 10,000 MWCO filters (Millipore). The purified dendrimer was lyophilized and partially carboxylated by reacting $1 \mathrm{mg}$ reconstituted PAMAM dendrimers with $0.308 \mathrm{mg}$ succinic anhydride in dimethyl sulfoxide (DMSO) overnight, corresponding to $70 \%$ conversion of surface amines to carboxyl groups. Partial carboxylation was verified by NMR spectroscopy (Figure S1). Additional detail for dendrimer modification can be found in reference 21 [Myung et al., Angew Chem Int Ed 2011, 50, 11769-72]. 
Supplementary information: Poellmann MJ et al, "Immunoavidity-Based Capture of Tumor Exosomes using Poly(amidoamine) Dendrimer Surfaces."

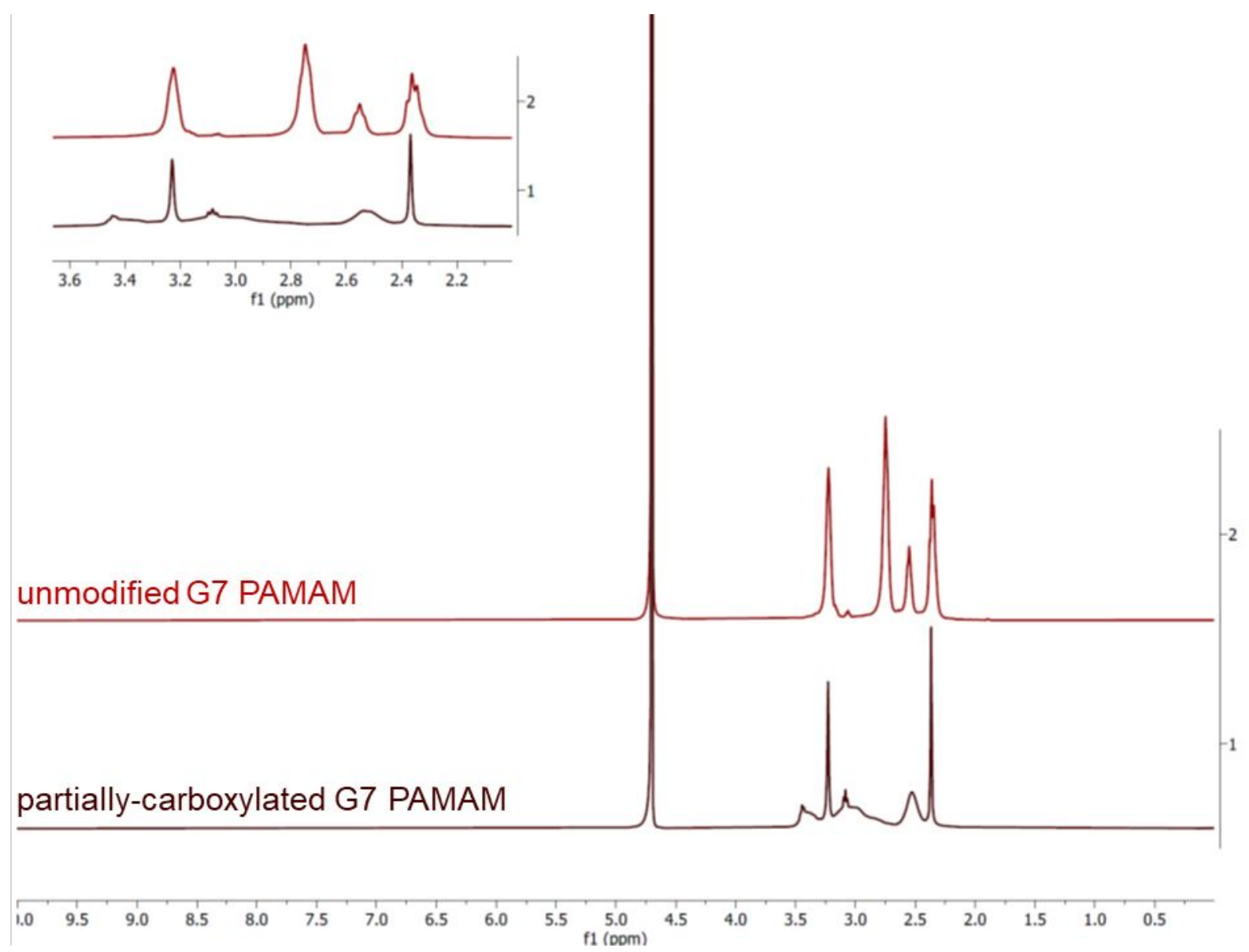

Figure S1. NMR spectrum of unmodified and partially carboxylated G7 PAMAM dendrimers.

\section{Surface Preparation}

Surface treatments were confined on epoxy-functionalized slides using silicone gaskets cast from polydimethylsiloxane with wells created using biopsy punches.

Preparation of full antibodies: Antibodies were prepared at $5 \mu \mathrm{g} \mathrm{mL}-1$ in $50 \mathrm{mM}$ 4-(2-hydroxyethyl)-1piperazineethanesulfonic acid buffer with $150 \mathrm{mM}$ sodium chloride (HBSS), pH 8. Antibody mixtures were prepared such that each antibody had a final concentration of $5 \mu \mathrm{g} \mathrm{mL} \mathrm{L}^{-1}$ in buffer. 
Supplementary information: Poellmann MJ et al, "Immunoavidity-Based Capture of Tumor Exosomes using Poly(amidoamine) Dendrimer Surfaces."

Preparation of reduced antibodies: Antibodies were prepared at a concentration of $0.5 \mu \mathrm{g} \mathrm{mL}^{-1}$ in PBS with 5 $\mathrm{mM}$ ethylenediaminetetraacetic acid (EDTA) and $3 \mathrm{mg} \mathrm{mL}^{-1}$ cysteamine $\mathrm{HCl}$. The solution was treated for 30 min at $37^{\circ} \mathrm{C}$, then purified using centrifugal filtration and a 10,000 MWCO filter (Amicon) immediately before surface conjugation. Antibodies were resuspended at a concentration of $5 \mu \mathrm{g} \mathrm{mL} \mathrm{L}^{-1}$ in PBS with EDTA.

Functionalization of glass surfaces: Epoxide-functionalized glass surfaces were rinsed with DDI water, incubated 30 min with $2 \%$ Tween-20, and treated overnight with full or reduced antibodies. Both types of antibodies formed a covalent bond to epoxide groups via free amines. Surfaces were blocked with $0.5 \%$ bovine serum albumin (BSA) for $1 \mathrm{~h}$ prior to exosome capture experiments. The combination of Tween-20 and BSA effectively mitigates nonspecific binding [Hong S et al., Langmuir 2007, 23, 12261-12268].

Preparation and functionalization of PEG surfaces: Capture surfaces consisting of antibodies tethered to glass by PEG were treated overnight with a mixture of $0.48 \mathrm{mg} \mathrm{mL}^{-1} 2000$ Da methoxy-PEG-amine (mPEG) and $0.02 \mathrm{mg}$ $\mathrm{mL}^{-1} 5000 \mathrm{Da}$ carboxy-PEG-amine in $1.5 \mathrm{M}$ potassium phosphate, $\mathrm{pH}$ 11. Surfaces were rinsed with DDI water and incubated 30 min with 2 vol.\% Tween-20. Surfaces were then rinsed and activated for 30 min with $15 \mathrm{mM}$ 1-ethyl-3-(3-dimethylaminopropyl)carbodiimide hydrochloride (EDC) and $25 \mathrm{mM} \mathrm{N}$-hydroxysuccinimide (NHS) in $100 \mathrm{mM}$ 2-(N-morpholino)ethanesulfonic acid buffer (MES), pH 5, with $150 \mathrm{mM}$ sodium chloride. The surfaces were then incubated with capture antibodies (full or reduced, see below) overnight at $4{ }^{\circ} \mathrm{C}$. Surfaces were blocked with $0.5 \%$ BSA for $1 \mathrm{~h}$ prior to exosome capture experiments.

Preparation and functionalization of single layer dendrimer surfaces with full antibodies: Capture surfaces consisting of PEG tethers, a single layer of partially carboxylated G7 PAMAM dendrimers, and full antibodies were treated overnight with a mixture of $0.48 \mathrm{mg} \mathrm{mL}^{-1} 2 \mathrm{kDa}$ methoxy-PEG-amine (mPEG) and $0.02 \mathrm{mg} \mathrm{mL}^{-1} 5$ $\mathrm{kDa}$ carboxy-PEG-amine in $1.5 \mathrm{M}$ potassium phosphate, $\mathrm{pH}$ 11. The carboxy-PEG was activated for $1 \mathrm{~h}$ with 
Supplementary information: Poellmann MJ et al, "Immunoavidity-Based Capture of Tumor Exosomes using Poly(amidoamine) Dendrimer Surfaces."

EDC and NHS before incubation overnight with $0.1 \mathrm{mg} \mathrm{mL}^{-1}$ partially carboxylated PAMAM dendrimer in HBSS. Surfaces were rinsed with DDI water and incubated 30 min with 2 vol.\% Tween-20. Surfaces were then rinsed and activated for 30 min EDC and NHS in MES buffer. Activated surfaces were incubated with full antibodies overnight at $4{ }^{\circ} \mathrm{C}$. Finally, surfaces were blocked with $0.5 \%$ BSA for $1 \mathrm{~h}$ prior to exosome capture experiments.

Preparation and functionalization of single layer dendrimer surfaces with reduced antibodies: Single layer surfaces were prepared as described above, but activated with $0.5 \mathrm{mg} \mathrm{mL}^{-1}$ water-soluble SMCC in PBS with EDTA for 30 min prior to adding reduced antibodies. Surfaces were blocked with $0.5 \%$ BSA for $1 \mathrm{~h}$ prior to exosome capture experiments.

Preparation and functionalization of dual layer dendrimer surfaces with full antibodies: Surfaces where treated overnight with $0.1 \mathrm{mg} \mathrm{mL}^{-1}$ partially carboxylated G7 PAMAM dendrimers in $1.5 \mathrm{M}$ potassium phosphate, $\mathrm{pH}$ 11. The surfaces were rinsed, activated with EDC and NHS, and incubated with $0.48 \mathrm{mg} \mathrm{mL}^{-1} \mathrm{mPEG}, 0.01 \mathrm{mg}$ $\mathrm{mL}^{-1} 5 \mathrm{kDa}$ carboxy-PEG-amine, and $0.01 \mathrm{mg} \mathrm{mL}^{-1} 20 \mathrm{kDa}$ carboxy-PEG-amine in HBSS for at least $4 \mathrm{~h}$. The surface was activated a second time with EDC and NHS in MES and treated overnight with $0.1 \mathrm{mg} \mathrm{mL}^{-1}$ partially carboxylated dendrimers in HBSS. The surfaces were rinsed, treated with Tween-20, activated with EDC and NHS, and incubated with full antibodies overnight at $4{ }^{\circ} \mathrm{C}$. Surfaces were blocked with $0.5 \%$ BSA for 1 $\mathrm{h}$ prior to exosome capture experiments.

Preparation and functionalization of dual layer dendrimer surfaces with reduced antibodies: Surfaces were prepared as described above for dual layer dendrimers. Surfaces were activated with SMCC as described above and incubated overnight with partially reduced antibodies at $4{ }^{\circ} \mathrm{C}$. Surfaces were blocked with $0.5 \%$ BSA for $1 \mathrm{~h}$ prior to exosome capture experiments. 
Supplementary information: Poellmann MJ et al, "Immunoavidity-Based Capture of Tumor Exosomes using Poly(amidoamine) Dendrimer Surfaces."

\section{Contact Angle Measurements}

Advancing and receding contact angles on modified slides were recorded with a Dataphysics OCA 15 Plus device with SCA 20 software (Filderstadt, Germany) using the sessile drop method. Measurements were collected using $8 \mu \mathrm{l}$ droplets of DDI water ejected at $1 \mu \mathrm{l} \mathrm{s}{ }^{-1}$. A total of 8 samples were tested in contact angle measurements.

The sessile drop contact angle technique was used to quantify the relative hydrophilicity of various prepared surfaces and verify polymer conjugation. The contact angles refer to the angle between the droplet-air and droplet-surfaces interfaces after the droplet was ejected (advancing, Figure 2a) and after a majority of the droplet was retracted (receding, Figure 2b). Measurements were used to verify enhanced conjugation of mPEG in a high-salt buffer compared to DDI water (Figure 1c,d). Figure 1e shows the advancing contact angle results that complement receding contact angle measurements reported in the main text.

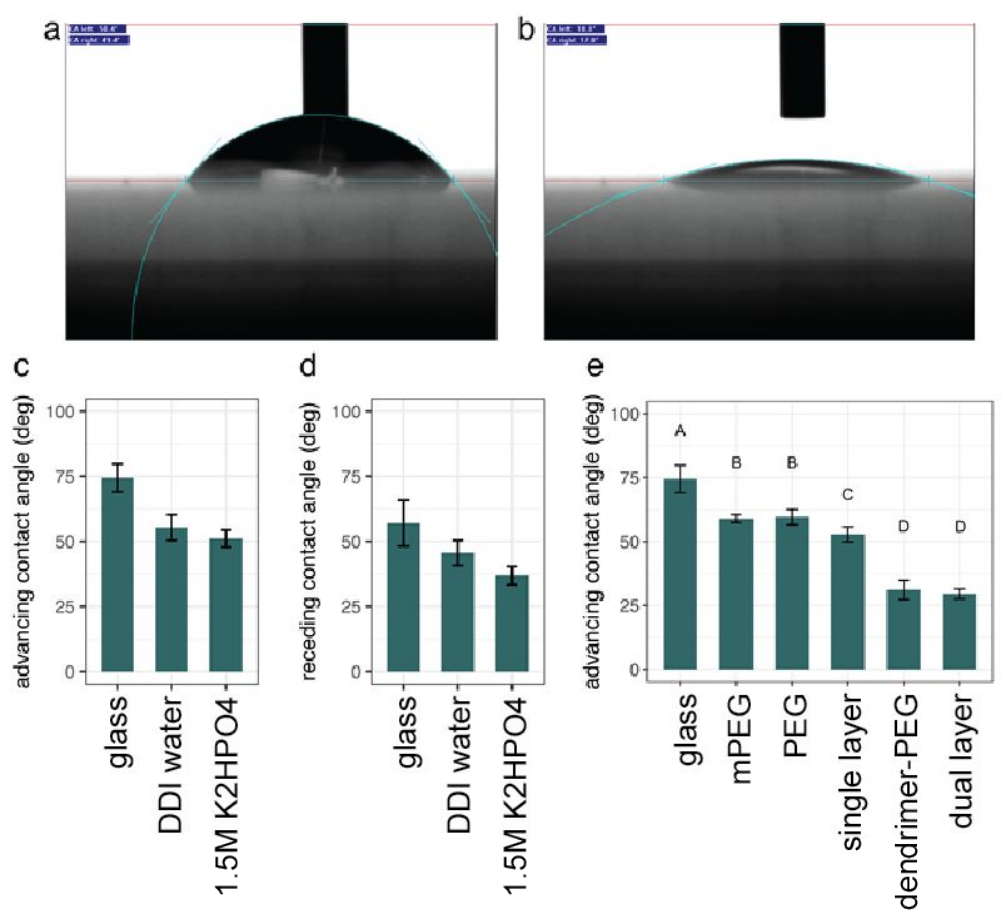


Supplementary information: Poellmann MJ et al, "Immunoavidity-Based Capture of Tumor Exosomes using Poly(amidoamine) Dendrimer Surfaces."

Figure S2. Contact angle results. Images of water droplets at the time of collecting a) advancing and b) receding contact angle measurements. c) The advancing and d) receding contact angles on glass are greater than mPEG controls, indicating successful conjugation of the polymer. A lower contact angle was observed when mPEG was conjugated in a high salt buffer, indicating a denser brush. e) Advancing contact angle measurements complimentary to, and show the same statistical groupings, as the results in Figure 2a. Charts show mean of $\mathrm{n}=$ $8+/$ - standard deviation, capital letters in (e) indicate statistical groupings. 
Supplementary information: Poellmann MJ et al, "Immunoavidity-Based Capture of Tumor Exosomes using Poly(amidoamine) Dendrimer Surfaces."

\section{Roughness Measurements}

Height profiles for roughness quantification were collected from polymer-modified slides with sharp, silicon nitride probes (Bruker SNL) using an Asylum Infinity Bio system (Oxford Instruments, Santa Barbara, CA). Measurements of samples hydrated with PBS were collected in tapping (AC) mode. Roughness was quantified as the Rq value (root mean square) of a $500 \mathrm{~nm}$ square image. Representative height scans are shown in Figure S3. A total of three independently fabricated surfaces were imaged to calculate roughness.

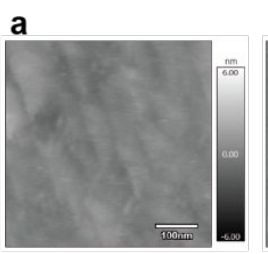

b
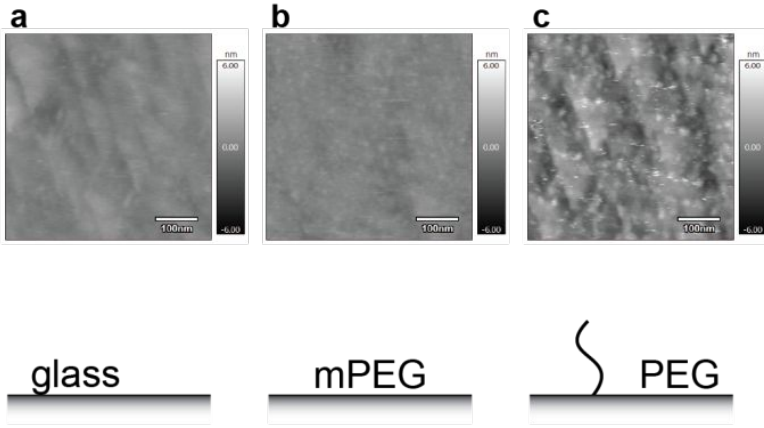

d
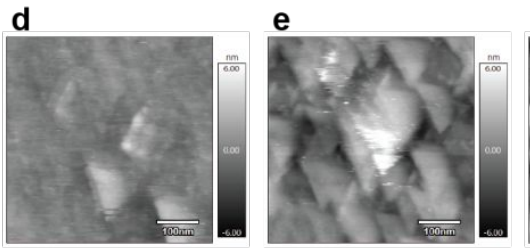

f
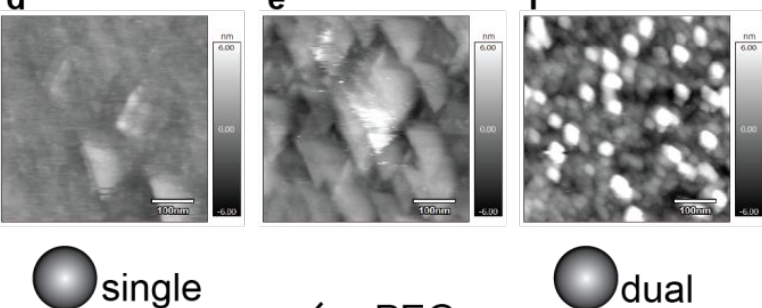
layer dendrimer dual layer dendrimer

Figure S3. Representative height profiles of polymer-coated surfaces. a) epoxide-functionalized glass, b) mPEGcoated glass, c) PEG surfaces, d) single layer dendrimer surfaces, e) surfaces coated with dendrimer followed by PEG tethers, and $\mathrm{f}$ ) dual layer dendrimer surfaces.

\section{Antibody Density Quantification}

Antibody density was estimated using fluorescently-labeled aEpCAM. The antibody was dissolved at $0.1 \mathrm{mg}$ $\mathrm{mL}^{-1}$ and treated with 1000x molar excess NHS-rhodamine (Thermo Fisher) for $30 \mathrm{~min}$, followed by partial reduction with cysteamine as described above. The fluorescently-labeled antibody was purified twice using 10,000 MWCO centrifugal filtration. Single layer and dual layer dendrimer surfaces were prepared as described 
Supplementary information: Poellmann MJ et al, "Immunoavidity-Based Capture of Tumor Exosomes using Poly(amidoamine) Dendrimer Surfaces."

above and activated with SMCC. Glass surfaces were used as controls, although the amine-reactive fluorescent dye precluded chemical conjugation to epoxide groups. , All surfaces were incubated overnight with $80 \mu \mathrm{l}$ of 5 $\mu \mathrm{mL}-1$ labeled antibody at $4{ }^{\circ} \mathrm{C}$. Finally, all surfaces were rinsed three times with PBS. Images of each well were collected using a Zeiss Axiovert inverted fluorescent microscope with automated scanning stage. Tiled images were collected at 10x magnification with consistent $1000 \mathrm{~ms}$ exposure for each experiment. Brightness and contrast manipulations completed in Zeiss ZEN software were consistent across all samples. Full-resolution tiff images covering 6000 pixels x 6000 pixels (corresponding to $2.7 \mathrm{~mm}$ x $2.7 \mathrm{~mm}$ ) in the center of each well were exported, avoiding nonspecific aggregation at the corners of each well. Fluorescent intensity was quantified from the mean greyscale value of each image reported NIH ImageJ software. A standard curve of antibody incubated, but not rinsed, over each well was employed to estimate coverage density, assuming each reduced antibody had a molecular weight of $75 \mathrm{kDa}$ and one active binding site. Figure S4 shows a mean of 1129 binding sites per square micron on dual layer dendrimer surfaces, which should be sufficient for multivalent binding of a $100 \mathrm{~nm}$-diameter vesicle. Single layer dendrimers had a mean of 1032 binding sites per square micron, which did not reach statistical significance $(\mathrm{p}=0.110)$.

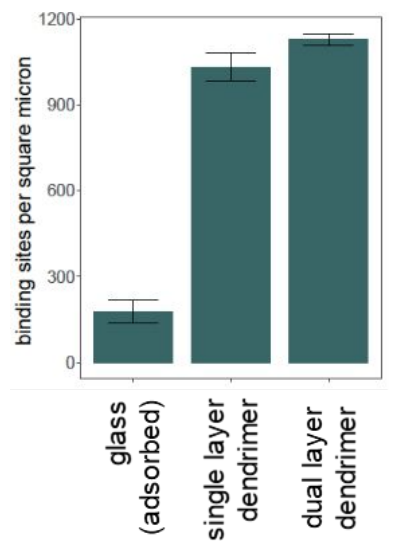

Figure S4. Estimated binding site density on single and dual layer dendrimer surfaces estimated with rhodamine-labeled aEpCAM. 
Supplementary information: Poellmann MJ et al, "Immunoavidity-Based Capture of Tumor Exosomes using Poly(amidoamine) Dendrimer Surfaces."

\section{Cell Culture and In Vitro Exosome Isolation}

The human metastatic breast cancer line MDA-MB-231 (ATCC, Manassas, VA) was expanded in L15 Medium (Corning, Tewksbury, MA) supplemented with 10 vol.\% fetal bovine serum (Corning) and 1 vol.\% penicillinstreptomycin (Corning) in humidified, $37^{\circ} \mathrm{C}$ incubators with atmospheric oxygen. The human epithelial breast cancer line MCF-7 (ATCC) was expanded in high glucose DMEM with sodium pyruvate (Corning) supplemented with 10 vol.\% fetal bovine serum and 1 vol.\% penicillin-streptomycin in humidified, $37{ }^{\circ} \mathrm{C}$ incubators with atmospheric oxygen supplemented with $5 \% \mathrm{CO}_{2}$. All cells were passaged using $0.25 \%$ trypsinEDTA (Corning) and seeded at 50-80\% confluence in 4 T-175 flasks for at least $24 \mathrm{~h}$ before adding serum-free medium containing $1 \mathrm{wt} . \%$ BSA. The conditioned medium was removed after 3 days and centrifuged at $300 \mathrm{xg}$, then $12,000 \mathrm{xg}$ to remove cells and other large debris. The resulting supernatant was passed through a $0.2 \mu \mathrm{m}$ membrane filter, concentrated to $1 / 3^{\text {rd }}$ the original volume, and ultracentrifuged at $120,000 \mathrm{xg}$ using a Beckman Type 45Ti rotor. The collected pellet was washed with PBS and centrifuged again before resuspending in PBS for storage at $-80^{\circ} \mathrm{C}$. Exosome concentration and size was determined by Nanoparticle Tracking Analysis (NTA, Malvern Nanosight NS300).

Table S1. Properties of exosomes isolated from conditioned media determined by NTA

\begin{tabular}{|l|c|c|c|c|}
\hline Cell type and batch & $\begin{array}{c}\text { concentration mean } \\
\left(\mathrm{mL}^{-1} \text { in } 0.1 \mathrm{~mL}\right)\end{array}$ & concentration SD & $\begin{array}{c}\text { size mean } \\
(\mathrm{nm})\end{array}$ & $\begin{array}{c}\text { size SD } \\
(\mathrm{nm})\end{array}$ \\
\hline MDA-MB-231 batch 1 & $1.24 \mathrm{E}+11$ & $2.34 \mathrm{E}+09$ & 116.9 & 1.9 \\
\hline MDA-MB-231 batch 2 & $1.52 \mathrm{E}+11$ & $3.62 \mathrm{E}+09$ & 141.2 & 15.5 \\
\hline MDA-MB-231 batch 3 & $9.50 \mathrm{E}+10$ & $3.35 \mathrm{E}+09$ & 118.8 & 5.9 \\
\hline MDA-MB-231 batch 4 & $2.19 \mathrm{E}+11$ & $6.15 \mathrm{E}+09$ & 135.9 & 6.6 \\
\hline MDA-MB-231 batch 5 & $1.46 \mathrm{E}+11$ & $3.59 \mathrm{E}+09$ & 114.8 & 7.4 \\
\hline MCF-7 batch 1 & $1.30 \mathrm{E}+11$ & $3.11 \mathrm{E}+09$ & 153.5 & 9.6 \\
\hline
\end{tabular}


Supplementary information: Poellmann MJ et al, "Immunoavidity-Based Capture of Tumor Exosomes using Poly(amidoamine) Dendrimer Surfaces."

\begin{tabular}{|l|c|c|c|c|}
\hline MCF-7 batch 2 & $1.17 \mathrm{E}+12$ & $2.04 \mathrm{E}+10$ & 134.8 & 4.7 \\
\hline MCF-7 batch 3 & $7.55 \mathrm{E}+11$ & $1.32 \mathrm{E}+10$ & 131.9 & 2.6 \\
\hline MCF-7 batch 4 & $8.05 \mathrm{E}+11$ & $1.81 \mathrm{E}+10$ & 129 & 3.1 \\
\hline MCF-7 batch 5 & $1.50 \mathrm{E}+12$ & $2.27 \mathrm{E}+10$ & 131.9 & 7.8 \\
\hline MCF-7 batch 6 & $1.07 \mathrm{E}+12$ & $2.10 \mathrm{E}+10$ & 124.9 & 6.1 \\
\hline
\end{tabular}

Western blotting was used to verify the presence of exosome-specific markers within the vesicles following ultracentrifugation (Figure S5). Exosomes were lysed by mixing with an equal volume 2x radioimmunoprecipitation assay buffer $(150 \mathrm{mM} \mathrm{NaCl}, 1 \% \mathrm{NP}-40,1 \%$ sodium deoxycholate, $0.1 \% \mathrm{SDS}, 50 \mathrm{mM}$ Tris-HCl, 2 mM EDTA, pH 7.5) containing protease inhibitors (Protease Inhibitor Cocktail II, Sigma-Aldrich). $15 \mu \mathrm{g}$ of total protein from the lysed exosomes were separated through SDS-PAGE and transferred to a PVDF membrane. Blots showed the presence of the chaperone HSP70 and tetraspanins CD63 and CD81 in exosomes collected from both cell types. Exosomes derived from MCF-7 cells additionally expressed CD9.
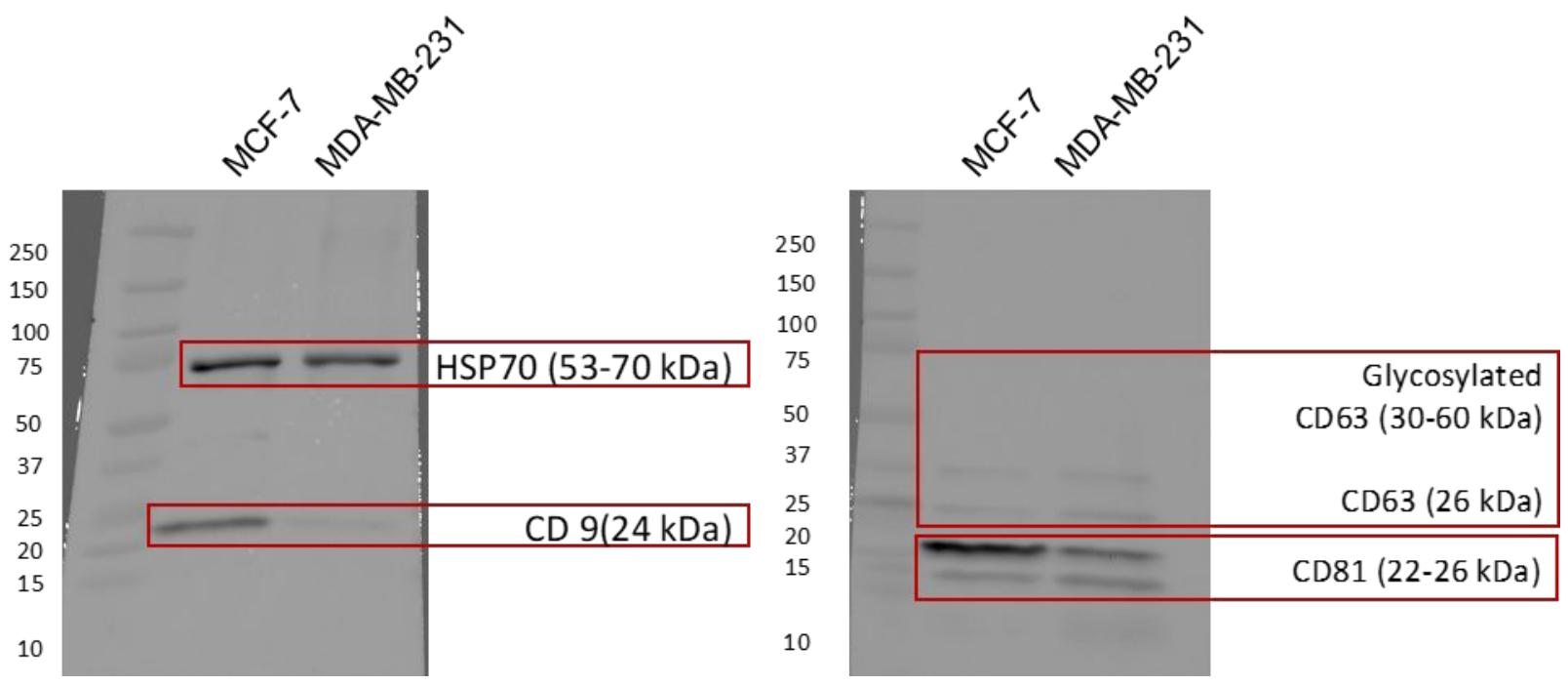
Supplementary information: Poellmann MJ et al, "Immunoavidity-Based Capture of Tumor Exosomes using Poly(amidoamine) Dendrimer Surfaces."

Figure S5. Western blotting confirmed the presence of tetraspanins CD81 and CD63 that differentiate exosomes from similarly-sized exosomes in MCF-7 and MDA-MB-231-derived exosomes.

\section{Capture and Quantification of In Vitro Exosomes}

Slides with silicone gaskets containing $6 \mathrm{~mm}$-diameter wells were functionalized as described above and coated with full or reduced antibody mixtures with a nominal concentration of $5 \mu \mathrm{g} \mathrm{mL}-1$ for each antibody. Exosomes isolated from conditioned media (Table S1) were resuspended at $5 \times 10^{9} \mathrm{~mL}^{-1}$ and treated 30 min at $37^{\circ} \mathrm{C}$ with 5 $\mu \mathrm{g} \mathrm{mL} \mathrm{m}^{-1} \mathrm{Vybrant} \mathrm{DiO}$ (Thermo) for $30 \mathrm{~min}$. MCF-7 exosomes used to compare surface configurations (Figure 3b) were diluted 1:1 in healthy human serum (Sigma) to a final concentration of $2.5 \times 10^{9} \mathrm{~mL}^{-1}$. MDA-MB-231 exosomes were labeled at a concentration of $2 \times 10^{10} \mathrm{~mL}^{-1}$, diluted $1: 1$ in healthy human serum, and then diluted further in serum. Concentrations depicted in Figure 3c were normalized assuming a $6 \mathrm{~mm}$-diameter well and $0.08 \mathrm{~mL}$ solution. All samples were placed on a rotary shaker and incubated for $4 \mathrm{~h}$ at room temperature. Wells were gently rinsed with PBS and placed on a Zeiss Axiovert inverted fluorescent microscope with automated scanning stage. Tiled images were collected at 10x magnification with consistent exposure time for each experiment. Brightness and contrast manipulations completed in Zeiss ZEN software were consistent across all samples in a given experiment. Full-resolution tiff images covering 6000 pixels $\mathrm{x} 6000$ pixels (corresponding to $2.7 \mathrm{~mm} \times 2.7 \mathrm{~mm}$ ) in the center of each well were exported, which avoided nonspecific aggregation and unrinsed material at the corners of each well. Fluorescent intensity was quantified from the mean greyscale value of each image reported NIH ImageJ software. 
Supplementary information: Poellmann MJ et al, "Immunoavidity-Based Capture of Tumor Exosomes using Poly(amidoamine) Dendrimer Surfaces."

\section{Capture and Quantification of Exosomes in Donor Blood Samples}

Blood samples were collected from three healthy human donors and five patients with diagnosed head and neck squamous cell carcinoma (HNSCC) at UW Health University Hospital in accordance with protocols approved by the University of Wisconsin-Madison Institutional Review Board (Table S2). Peripheral blood was collected from each donor in a pair of sodium heparin tubes. Within $2 \mathrm{~h}$ of collection, $10 \mathrm{~mL}$ peripheral blood was diluted with $10 \mathrm{~mL}$ PBS and layered on top of Ficoll-Paque Plus (GE Life Sciences) for gradient separation. Approximately $10 \mathrm{~mL}$ of the top (plasma) layer was frozen and stored at $-80^{\circ} \mathrm{C}$.

Plasma samples were thawed and treated with Vybrant $\mathrm{DiO}$ at $5 \mu \mathrm{mL} \mathrm{m}^{-1}$ for $30 \mathrm{~min}$ at $37^{\circ} \mathrm{C}$ to label all vesicular material with green fluorescent dye. The samples were centrifuged at $1000 \mathrm{xg}$ for 10 minutes to remove any large aggregates from solution, then incubated over capture surfaces functionalized with reduced aEpCAM, aEGFR, and aHER2 for $4 \mathrm{~h}$ at room temperature with agitation. Exosome capture was quantified as described above.

Table S2. Demographic information and disease status of HNSCC patients and healthy donors

\begin{tabular}{|l|c|c|c|}
\hline Donor code & age & sex & tumor stage \\
\hline $18098-04$ & 70 & $\mathrm{M}$ & stage I (cT1, cN1, cM0) \\
\hline $18098-05$ & $>90$ & $\mathrm{~F}$ & stage IVA (cT4a, NC2c, cM0) \\
\hline $18098-06$ & 70 & $\mathrm{~F}$ & stage II (cT2, cN0, cM0) \\
\hline $18098-07$ & 49 & $\mathrm{M}$ & stage IVA (cT3, cN2b, cM0) \\
\hline $18098-08$ & 41 & $\mathrm{M}$ & stage IVA (cT2, cN2b, cM0) \\
\hline healthy donor 3 & 26 & $\mathrm{~F}$ & $\mathrm{NA}$ \\
\hline healthy donor 4 & 20 & $\mathrm{M}$ & $\mathrm{NA}$ \\
\hline healthy donor 5 & 20 & $\mathrm{M}$ & $\mathrm{NA}$ \\
\hline
\end{tabular}


Supplementary information: Poellmann MJ et al, "Immunoavidity-Based Capture of Tumor Exosomes using Poly(amidoamine) Dendrimer Surfaces."

\section{AFM Force Spectroscopy}

Capture surfaces were prepared with a EpCAM at $5 \mu \mathrm{g} \mathrm{mL}-1$. A single gold-coated silicon nitride probe (Asylum TR400PB) was treated with a mixture of $0.1 \mathrm{mg} \mathrm{mL}^{-1} 7.5 \mathrm{kDa}$ carboxyl-PEG-thiol (JenKem) and $1.9 \mathrm{mg} \mathrm{mL}^{-1} 5$ kDa methoxy-PEG-thiol (JenKem) in DDI water for $1 \mathrm{~h}$. Four surfaces were tested: PEG without antibodies, PEG with full antibodies, nonfunctionalized dual layer dendrimers, and dual layer dendrimers functionalized with partially reduced antibodies. The probes were activated with EDC and NHS before adding recombinant human EpCAM-Fc chimera (R\&D Systems) at $5 \mu \mathrm{g} \mathrm{mL} \mathrm{m}^{-1}$ overnight at $4{ }^{\circ} \mathrm{C}$. Optical lever sensitivity was determined by indenting on uncoated glass, and spring constant determined by the thermal noise method. The spring constant was determined to be $6.85 \mathrm{pN} \mathrm{nm}^{-1}$.

Experiments were conducted using an Asylum Infinity Bio system (Oxford Instruments) with probe and sample submerged in PBS. Each surface was engaged in at least 5 discrete locations with approximately 15 force curves collected at each. Force curves consisted of a $1 \mu \mathrm{m}$ approach at $500 \mathrm{~nm} \mathrm{~s}^{-1}, 5 \mathrm{~s}$ dwell with the cantilever in light contact with the surface $(0.2 \mathrm{~V}$ setpoint $)$, and retract velocity at $500 \mathrm{~nm} \mathrm{~s}^{-1}$. Data collection was at $2 \mathrm{kHz}$.

Data were analyzed with a semi-automated algorithm programmed in $\mathrm{R}$ using the Shiny package. The workflow is summarized in Figure S6. Briefly, the wave file produced by Asylum instrumentation was read with the aid of the IgorR package in R. The waves were automatically segmented, filtered, corrected for virtual deflection, and corrected such that the contact point was zero force and zero height. Individual events were identified as abrupt changes in deflection (minimum $5 \mathrm{pN} \mathrm{ms}^{-1}$ ) with minimum magnitude (16 $\left.\mathrm{pN}\right)$. 
Supplementary information: Poellmann MJ et al, "Immunoavidity-Based Capture of Tumor Exosomes using Poly(amidoamine) Dendrimer Surfaces."
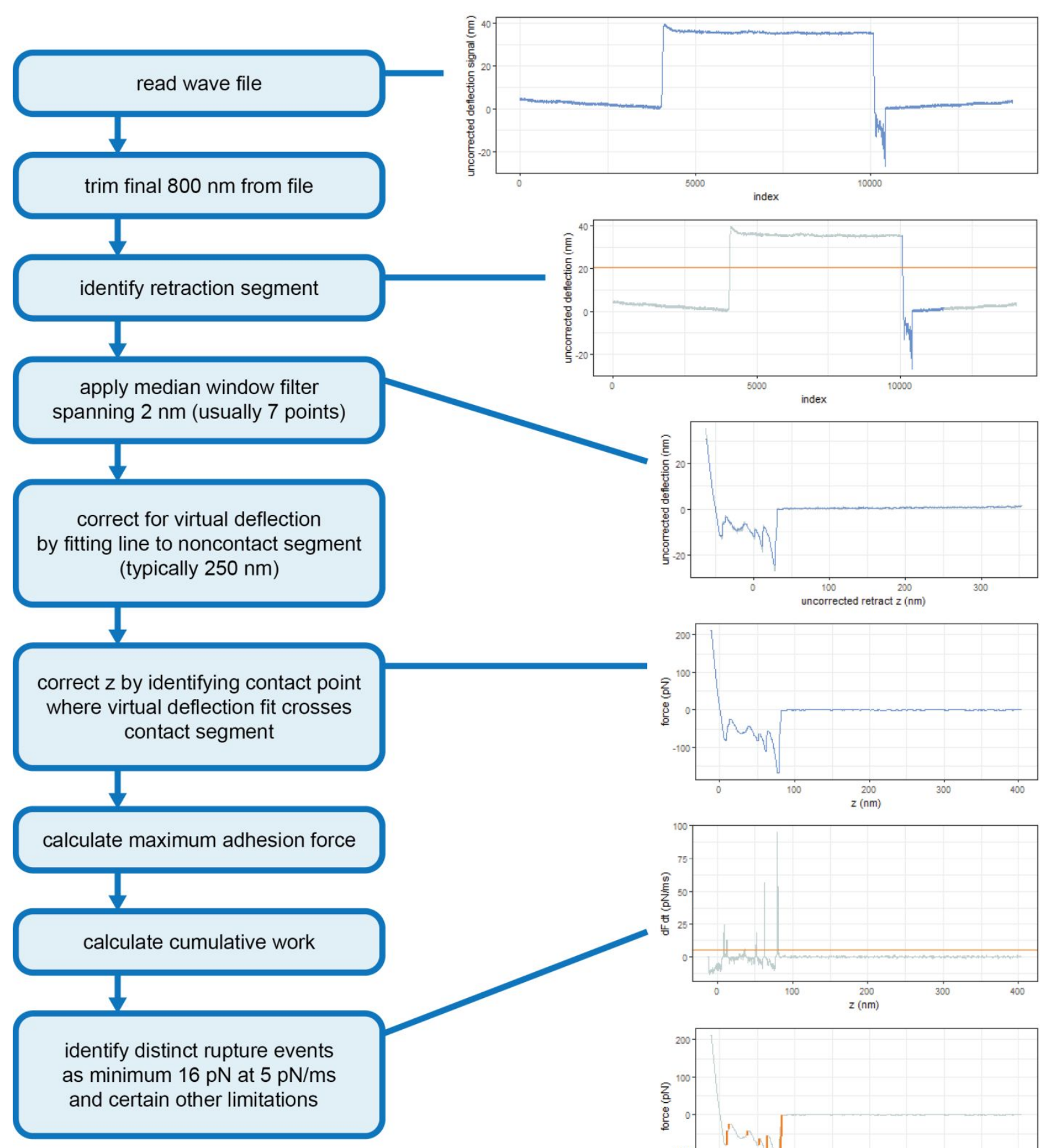

correct $z$ by identifying contact point where virtual deflection fit crosses contact segment
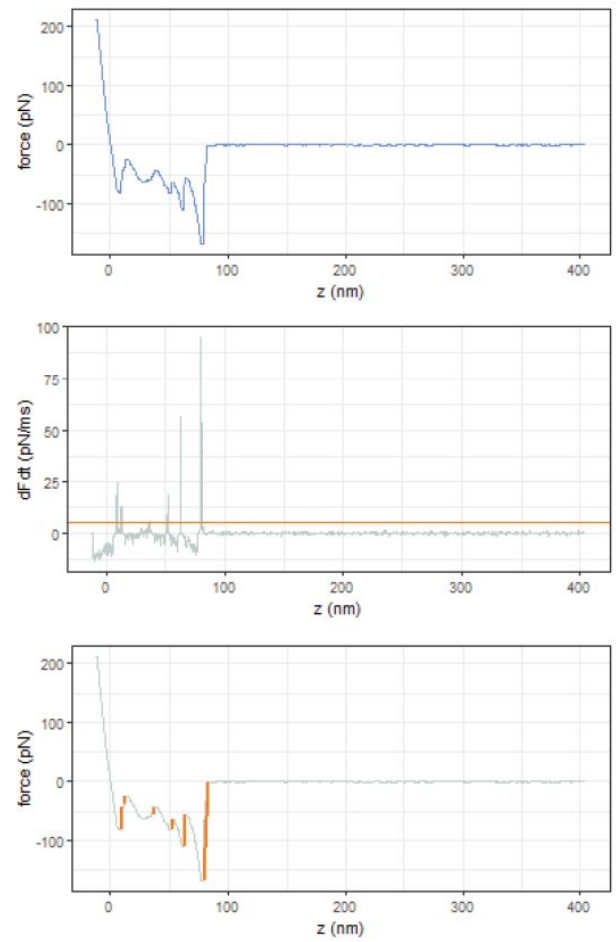

Figure S6. AFM force spectroscopy analysis workflow 
Supplementary information: Poellmann MJ et al, "Immunoavidity-Based Capture of Tumor Exosomes using Poly(amidoamine) Dendrimer Surfaces."

\section{Statistical Analysis}

Most assays were compared by one- or two-way analysis of variance with Tukey post-hoc means testing using $\mathrm{R}$ software, assuming $\mathrm{p}<.05$ to be significant. Force spectroscopy results were compared with Kruskal-Wallace tests because the data were not normally distributed, with pairwise comparisons by Nemenyi post-hoc tests from the R package "PMCMR." All charts were created in R with the "ggplot2" package. 